Julia Maria MATERA ${ }^{1}$

Angélica Cecília

TATARUNAS ${ }^{1}$

Renata Moris Domenico

OLIVEIRA $^{1}$

Milena BRUGNARO²

Renata Ferri MACCHIONE²

Correspondência para:

Rua Orlando Marques dePaiva, 87 -Butantã

Cidade Universitária, 05508900 - São

Paulo, SP - email: materajm@usp.br

Recebido para publicação: 16/05/2006 Aprovado para publicação: 23/08/2007

\title{
Estudo epidemiológico retrospectivo de cães portadores de ruptura do ligamento cruzado cranial: 323 casos (1999 a 2005)
}

\author{
1 Departamento de Cirurgia da Faculdade de Medicina Veterinária e Zootecnia da \\ Universidade de São Paulo, São Paulo-SP \\ 2 Médica Veterinária Autônoma
}

\section{Resumo}

O objetivo do presente trabalho foi caracterizar a população de cães portadores de ruptura do ligamento cruzado cranial (RLCC) para fatores considerados de risco como raça, idade, sexo e peso corporal. A base de dados consistiu de informações resgatadas de prontuários de 323 cães com diagnóstico de ruptura de ligamento cruzado cranial atendidos no Serviço de Cirurgia de Pequenos Animais do Hospital Veterinário da Faculdade de Medicina Veterinária e Zootecnia da Universidade de São Paulo em um período de 7 anos (1999 a 2005). Os resultados mostraram que as raças de maior prevalência foram o Leonberger (100\%), Cane Corso (66,7\%), Dogue de Bordeau (50\%), Starffordshire Terrier (40\%) e o Chow Chow (36\%), estando ainda o Rottweiler (11,6\%) e o Labrador Retriever $(8,1 \%)$ em $10^{\circ}$ e $13^{\circ}$ lugares, respectivamente. As idades de maior freqüência foram 2, 4, 3, 7 e 6 anos (média $=5,58$ anos). Fêmeas (59,14\%) foram mais acometidas do que machos $(40,86 \%)$ e animais inteiros $(76,15 \%)$ foram mais representativos do que animais castrados $(17,76 \%)$. Quanto ao peso, entre 6 e $15 \mathrm{~kg}(32,82 \%)$ seguido por animais entre 36 e $45 \mathrm{~kg}(17,03 \%)$. Pode-se observar que os dados em nosso meio tendem a se assemelhar com a literatura estrangeira: ocorrência da RLCC em animais mais jovens de raças de grande porte e gigantes, o Rottweiler como uma raça em comum a fêmea é mais representativa do que o macho.

\section{Introdução e Revisão de Literatura}

A ruptura do ligamento cruzado cranial (RLCC) é afecção freqüente que acomete a articulação do joelho no cão. Apesar de ter sido diagnosticada pela primeira vez em 1926, a causa da afecção, na maioria das vezes, ainda permanece obscura, bem como a melhor opção de tratamento. Em uma pequena porcentagem de cães a RLCC pode ser puramente traumática, os animais acometidos geralmente são jovens e ocorre avulsão da inserção ligamentar do osso. Na grande parte dos cães, porém, a doença é de curso crônico e não há um evento traumático distinto associado ao início do processo. ${ }^{1,2}$

Preocupados em caracterizar a população de risco para a RLCC, pesquisadores vêm tentando demonstrar, pelo estudo de amostras representativas de cães portadores da afecção, a sua prevalência em relação a fatores como idade, peso, sexo e padrão racial. $3,4,5,6$

Segundo literatura estrangeira, os animais acometidos geralmente possuem entre 5 e 7 anos. As fêmeas são mais prevalentes do que os machos e a castração contribui para o aparecimento da doença em ambos os sexos. Também tem se observado que cães de raças grandes tendem a apresentar a doença em idade mais precoce quando comparado a cães de raças pequenas e médias ${ }^{6,7,8}$, caracterizando a chamada doença do cruzado, na qual há degeneração progressiva do ligamento até a sua 
completa ruptura. ${ }^{3}$

O objetivo do presente trabalho foi caracterizar a população de cães portadores de RLCC, atendidos no Serviço de Cirurgia de Pequenos Animais do Hospital Veterinário da Faculdade de Medicina Veterinária e Zootecnia da Universidade de São Paulo (HOVET/FMVZ/USP) para fatores considerados de risco, como raça, idade, sexo e peso corporal.

\section{Material e Método}

Foram revisados os prontuários de 323 cães com diagnóstico de RLCC atendidos no Serviço de Cirurgia de Pequenos Animais do HOVET/FMVZ/ USP em um período de sete anos (1999 a 2005).

O relato do teste de gaveta positivo ${ }^{9}$ foi considerado diagnóstico para a afecção. Os cães com ausência de instabilidade articular detectável clinicamente ou resultado duvidoso foram incluídos nesta pesquisa quando a cirurgia confirmou o diagnóstico.

Os animais foram avaliados quanto à raça, idade, sexo, peso corporal e membro acometido. Os cães foram agrupados segundo o peso em $<5$; 6 a 15; 16 a 25 ;
26 a 35; 36 a 45 e $>46$ kg. Para o sexo considerou-se fêmeas inteiras, fêmeas castradas, machos inteiros e machos castrados.

Para o cálculo da prevalência do padrão racial (porcentagem relativa) dos animais portadores de RLCC, utilizou-se como população de risco o total de cães atendidos $(\mathrm{n}=8496)$ no Serviço de Cirurgia do HOVET/FMVZ/USP no período de janeiro de 2002 a dezembro de 2005. Este período diferiu daquele proposto para os cães portadores de RLCC (1999-2005) devido ao fato destes dados estarem acessíveis em arquivos informatizados e somarem uma amostra considerada satisfatória. A prevalência foi calculada para cada grupo racial dividindo-se o numero de cães do grupo com RLCC pelo numero de cães na população de risco correspondente e multiplicando-se por 100 .

O tipo de dieta foi estimado quanto à administração de ração comercial, caseira ou mista (associação de ambas). A causa relatada pelo proprietário foi classificada como sendo traumática, na presença de histórico de trauma significativo (exemplo: atropelamento) ou espontânea, durante atividade normal (exemplo: brincando, caminhando).

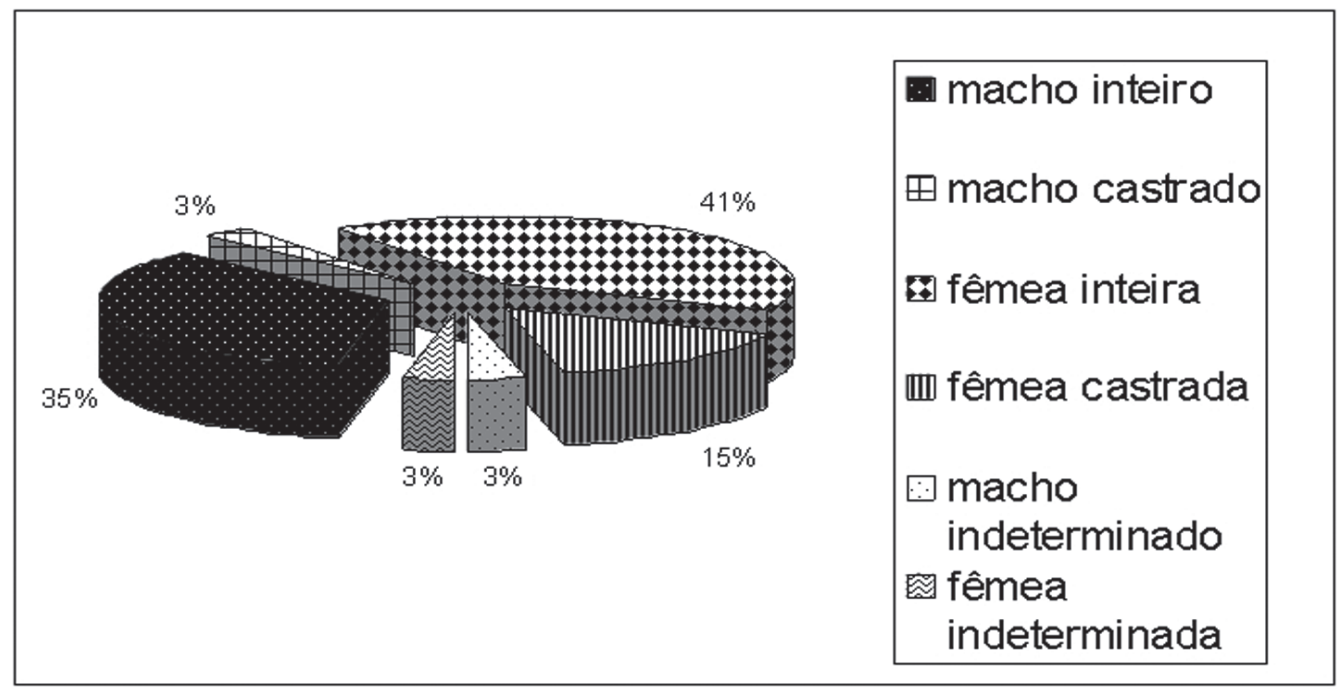

Figura 1 - Gráfico de distribuição dos 323 cães portadores de RLCC, segundo ao sexo: machos inteiros $(\mathrm{n}=112)$, machos castrados $(n=9)$, fêmeas inteiras $(n=134)$, fêmeas castradas $(n=48)$, machos indeterminados $(n=11)$ e fêmeas indeterminadas $(n=9)$ 
Tabela 1 - Dados referentes ao padrão racial dos 323 cães portadores de RLCC atendidos HOVET/FMVZ/USP no período de 1999 a 2005 em relação ao seu número absoluto, porcentagem absoluta e porcentagem relativa referente ao total de cães $(n=8496)$ atendidos neste mesmo local no período de 2002 a 2005. São Paulo, 2006

\begin{tabular}{|c|c|c|c|}
\hline Raça & $\begin{array}{l}\text { Número Absoluto } \\
(\mathrm{N})\end{array}$ & $\begin{array}{l}\text { Porcentagem } \\
\text { Absoluta (\%) }\end{array}$ & $\begin{array}{l}\text { Porcentagem } \\
\text { Relativa (\%) }\end{array}$ \\
\hline Leonberger & 1 & 0,6 & 100 \\
\hline Cane Corso & 2 & 0,6 & 66,7 \\
\hline Dogue de Bordeau & 1 & 0,6 & 50 \\
\hline American Starfford Terrier & 2 & 0,6 & 40 \\
\hline Chow-chow & 4 & 1,24 & 36 \\
\hline Mastin Napolitano & 4 & 1,24 & 28,58 \\
\hline Bulldog Inglês & 6 & 1,85 & 24 \\
\hline Pastor Canadense & 1 & 0,6 & 20 \\
\hline Mastiff Inglês & 2 & 0,6 & 14,28 \\
\hline Rottweiler & 50 & 15,48 & 11,16 \\
\hline Fila Brasileiro & 9 & 2,78 & 10,8 \\
\hline Pit bull & 17 & 5,27 & 8,13 \\
\hline Labrador & 21 & 6,51 & 8,1 \\
\hline Yorkshire & 9 & 2,78 & 7,3 \\
\hline Bichon Frisê & 2 & 0,6 & 6,67 \\
\hline Boxer & 19 & 5,88 & 6,6 \\
\hline Weimaraner & 3 & 0,91 & 6 \\
\hline Maltês & 3 & 0,91 & 5,8 \\
\hline São Bernardo & 1 & 0,6 & 5,56 \\
\hline Beagle & 4 & 1,24 & 4,8 \\
\hline Poodle & 56 & 17,34 & 4,34 \\
\hline Lhasa Apso & 3 & 0,91 & 4 \\
\hline Fox Paulistinha & 3 & 0,91 & 2,52 \\
\hline Golden Retriever & 1 & 0,6 & 2,2 \\
\hline Dogue Alemão & 1 & 0,6 & 1,54 \\
\hline Cocker Spaniel & 5 & 1,54 & 1,33 \\
\hline Pastor Belga & 1 & 0,6 & 1,33 \\
\hline Husky Siberiano & 1 & 0,6 & 1,25 \\
\hline Pinscher & 5 & 1,54 & 1,21 \\
\hline Doberman & 1 & 0,6 & 0,95 \\
\hline Bull Mastiff & 1 & 0,6 & 0,6 \\
\hline Pastor Alemão & 2 & 0,6 & 0,4 \\
\hline SRD & 82 & 25,37 & 0,1 \\
\hline Total & 323 & 100 & \\
\hline
\end{tabular}

SRD: Sem Raça Definida

\section{Resultados}

Os resultados referentes ao número absoluto, porcentagem e prevalência em relação ao padrão racial dos cães portadores de RLCC encontram-se na tabela 1. Os dados sobre o número absoluto e a porcentagem relativa à idade dos cães com RLCC estão dispostos na tabela 2. A média de idade dos animais portadores de RLCC foi de 5.58 anos.

Os dados pertinentes ao número absoluto e porcentagem entre machos, fêmeas, machos castrados e fêmeas castradas encontram-se na figura 1. Em 11 machos e nove fêmeas não foi possível determinar se eram castrados ou não.

Os valores referentes ao peso corpóreo dos animais estudados encontramse na figura 2. A RLCC ocorreu no joelho direito em 131 cães $(40,57 \%)$ e no joelho esquerdo em 152 (47,05\%), sendo bilateral em 40 animais $(12,38 \%)$. A causa foi espontânea em 310 cães $(95,75 \%)$ e traumática em 13 (4,25\%). Dentre os animais estudados, $207(67,18 \%)$ ingeriam ração 
Tabela 2 - Dados referentes ao número absoluto e porcentagem da idade dos 323 cães portadores de RLCC atendidos no HOVET/FMVZ/USP no período de 1999 a 2005. São Paulo, 2006

\begin{tabular}{ccc}
\hline Idade (Anos) & Número Absoluto $(\mathrm{N})$ & Porcentagem $(\%)$ \\
\hline$<1$ & 8 & 2,49 \\
1 & 29 & 8,97 \\
2 & 39 & 12,07 \\
3 & 33 & 10,22 \\
4 & 38 & 11,77 \\
5 & 17 & 5,27 \\
6 & 31 & 9,60 \\
7 & 36 & 11,14 \\
8 & 23 & 7,12 \\
9 & 23 & 7,12 \\
10 & 17 & 5,27 \\
11 & 9 & 2,79 \\
12 & 10 & 3,10 \\
13 & 7 & 2,17 \\
14 & 2 & 0,60 \\
15 & 1 & 0,30 \\
\hline Total & $\mathbf{3 2 3}$ & $\mathbf{1 0 0} \%$ \\
\hline
\end{tabular}

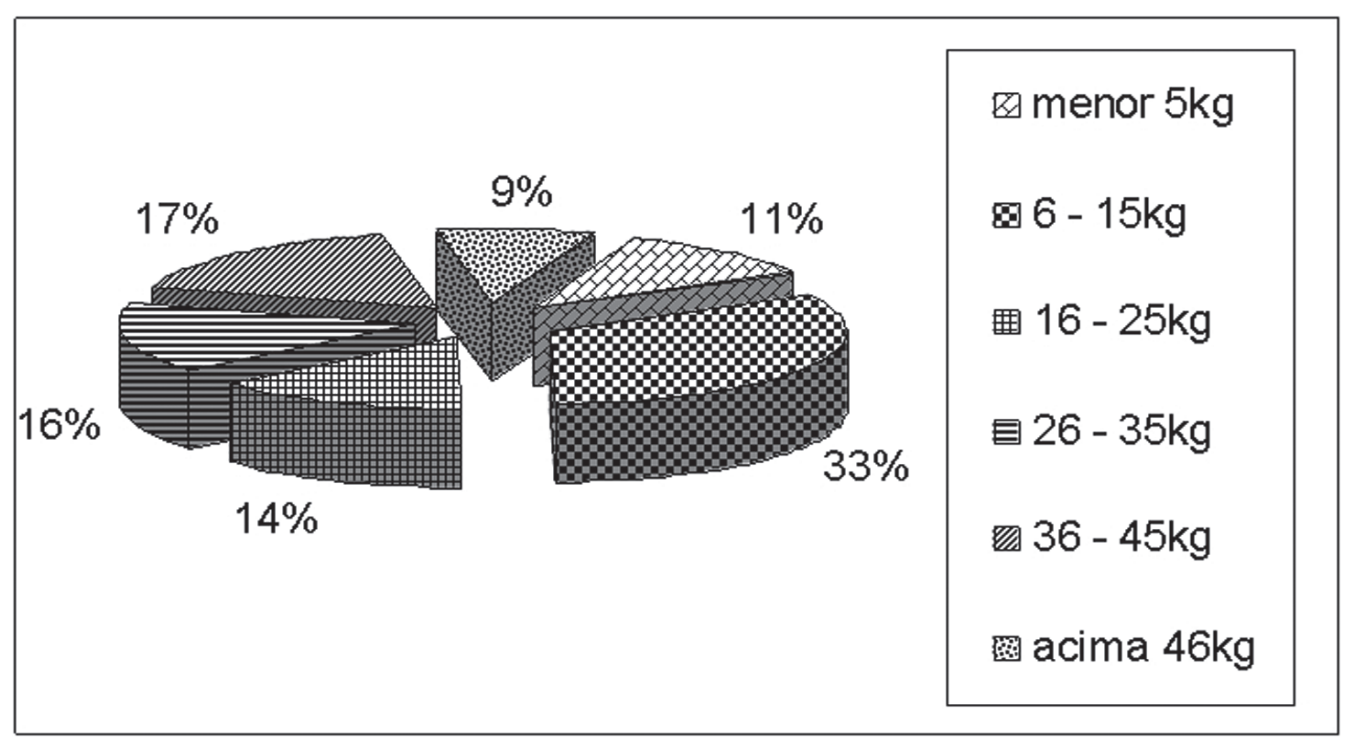

Figura 2 - Gráfico de distribuição dos 323 cães portadores de RLCC, segundo o peso corpóreo: $<5 \mathrm{Kg}(\mathrm{n}=37)$ , $6-15 \mathrm{Kg}(\mathrm{n}=106), 16-25 \mathrm{Kg}(\mathrm{n}=45), 26-35 \mathrm{Kg}(\mathrm{n}=52), 36-45 \mathrm{Kg}(\mathrm{n}=55),>46 \mathrm{Kg}(\mathrm{n}=28)$

comercial, $24(7,43 \%)$ comida caseira e 76 $(23,54 \%)$ uma dieta mista. Em seis $(1,85)$ animais o tipo de alimentação foi desconhecido.

\section{Discussão}

O prévio conhecimento dos fatores considerados de risco para uma determinada doença pode permitir um melhor entendimento da sua etiologia, bem como fornecer informações sobre a sua prevenção e diagnóstico. Duval et al. ${ }^{4}$ e Lampman, Lund e Lipowitz ${ }^{5}$ procuraram caracterizar os cães portadores de RLCC em relação à raça, sexo e peso corpóreo. Whitehair, Vasseur e Willits ${ }^{6}$ por sua vez fizeram estudo epidemiológico e avaliaram a prevalência da 
afecção para, além dos fatores acima, a idade, o tipo de dieta e a condição corpórea. Neste estudo procurou-se caracterizar os cães portadores de RLCC atendidos no HOVET / FMVZ / USP quanto aos fatores considerados de risco como raça, idade, sexo, peso e dieta. Também, a partir de uma base de dados deste mesmo hospital, calculou-se a prevalência para o padrão racial. Os dados oriundos deste trabalho foram comparados basicamente com a literatura americana, salientando-se que a carência de trabalhos semelhantes em nosso meio motivou a sua realização.

As lesões do ligamento cruzado cranial têm sido classificadas como traumáticas (agudas) ou degenerativas (crônicas). ${ }^{4}$ Enquanto a primeira acomete animais em crescimento e ocorre avulsão de uma das extremidades do ligamento ${ }^{10}$, na forma crônica a RLCC é espontânea, havendo um estiramento inicial, ruptura parcial e então total do ligamento ${ }^{3}$. Os casos aqui relatados referem-se à forma crônica em sua grande maioria $(95,75 \%)$, nos quais existe ausência de histórico de trauma significativo.

A RLCC, classicamente uma doença descrita em cães de meia idade e velhos ${ }^{11,12}$, nos quais se atribuía a injúria do ligamento a alterações degenerativas ocorridas em longo prazo, vem sendo relatada principalmente em cães jovens de grande porte ${ }^{3,6,13}$, os quais são atualmente considerados susceptíveis à afecção. ${ }^{6}$ Devido a esta relação entre idade e porte; e, conseqüentemente padrão racial, procuraremos avaliá-los conjuntamente.

Quanto aos quesitos idade e peso, Whitehair, Vasseur e Willits ${ }^{6}$ observaram que os cães com $>22 \mathrm{~kg}$ além de terem uma maior prevalência para a RLCC tenderam a romper o ligamento em idade mais precoce. Porém, estes autores também observaram, no computo geral dos 10.769 cães estudados, que a prevalência da RLCC aumentou conforme os cães tornaram-se mais velhos, sendo principalmente acometidos os animais entre 7 e 10 anos de idade. ${ }^{6} \mathrm{~A}$ força do ligamento cruzado cranial do cão declina com o envelhecimento devido à perda de organização dos feixes de fibras e alterações metaplásicas dos elementos celulares; e, nos cães de raças maiores, estas alterações pronunciem-se em idade mais precoce. ${ }^{13}$ Lampman, Lund e Lipowitz ${ }^{5}$, por sua vez, ao estudarem uma população bem menor de cães $(n=755)$ com RLCC obtiveram a idade média de 6,6 anos. Neste estudo a idade média foi 5,6 anos, sendo que $45,52 \%$ dos animais possuíam até 4 anos, estando próximo dos dados relatados por Lampman, Lund e Lipowitz ${ }^{5}$; Vasseur, Pool e Arnoczky ${ }^{11}$ e Bennett et al. 3. Também, no presente estudo, excluindo os cães sem raça definida, $50,6 \%$ dos cães acometidos são de raças de grande porte e gigantes, enquanto $33,6 \%$ e $15,8 \%$ correspondem aos cães de porte pequeno e médio, respectivamente, estando de acordo com os autores supracitados.

Ao se considerar a freqüência em que um determinado padrão racial apresentou a RLCC, torna-se difícil a comparação dos resultados deste estudo com a literatura estrangeira, pois várias raças consideradas predisponentes para a afecção, como por exemplo, o Newfoundland e Starffordshire Terrier, ${ }^{4,5,6}$ não são comuns em nosso meio. Também, raças brasileiras como o Fila Brasileiro e o Fox Paulistinha, que não são prováveis de serem encontradas nos trabalhos pesquisados foram aqui representadas por nove $(2,78 \%)$ e três $(0,91 \%)$ cães, respectivamente. Outro fator conflitante relaciona-se ao modismo da raça em certo local e período de tempo ${ }^{3}$, tal qual se creditou a freqüência da raça Pit Bull nesta pesquisa, a qual compreendeu $17(5,27 \%)$ animais e não foi citada nos trabalhos estudados.

Segundo Whitehair, Vasseur e Willits ${ }^{6}$; Duval et al. ${ }^{4}$ e Bennett et al. ${ }^{3}$, a RLCC ocorre mais em raças grandes do que em raças pequenas. Neste estudo as cinco raças de maior prevalência (freqüência relativa) em ordem decrescente foram o Leonberger, Cane Corso, Dogue de Bordeau, Starffordshire Terrier e o Chow Chow, concordando com os autores acima. Em concordância com os dados da literatura 
encontramos também maior freqüência relativa das raças Starffordshire Terrier e Chow Chow. ${ }^{4,5,6}$ As raças Leonberger, Cane Corso, Dogue de Bordeau, Starffordshire Terrier evidenciam-se pelo seu baixo índice representativo na população controle deste estudo, por serem pouco comuns em nosso país.

O Rottweiler e o Labrador Retriever, raças freqüentes em nosso meio, também são consideradas de risco para a RLCC segundo a literatura estrangeira. 3,4,6 Nesta pesquisa elas encontram-se em $10^{\circ}$ e $13^{\circ}$ colocação, respectivamente, portanto relevantes entre as 33 raças estudadas. Wingfield et al. ${ }^{12}$ realizaram estudo comparando as propriedades físicas do ligamento cruzado cranial do Rottweiler e do Greyhound e demonstraram que o ligamento do primeiro é mais vulnerável a lesão por requerer metade da carga por unidade de massa para a ruptura. Piermattei, Flo e Decamp ${ }^{8}$, por sua vez, sugerem um maior acometimento pela afecção nos últimos 15-20 anos em cães de membros pouco angulados, exemplificando com o Labrador Retriever.

Harasen $^{13}$, Barnes ${ }^{14}$, Gambardella, Wallace e Cassidy ${ }^{15}$ e Smith e Torg ${ }^{16}$ têm relatado maior incidência da RLCC em fêmeas do que em machos, fato constatado neste estudo. Porém, outros autores têm ainda inferido que animais castrados são mais susceptíveis a RLCC do que animais inteiros 4,6 e tal fato tem sido correlacionado com um estudo experimental realizado em ratas, nas quais a ovariectomia reduziu o conteúdo de elastina e o diâmetro das fibras da cápsula de articulação coxofemoral, justificando desta forma a ação dos hormônios sexuais no metabolismo do colágeno. ${ }^{17} \mathrm{Os}$ resultados deste estudo estão de acordo com os autores supracitados no que tange ao maior acometimento de fêmeas quando comparado aos machos, porém a RLCC foi mais freqüente em animais inteiros do que em animais castrados.

Lampman, Lund e Lipowitz ${ }^{5}$ avaliaram a relação do tipo de dieta com a RLCC no cão e concluíram que animais alimentados com dieta enriquecida e, conseqüentemente maior massa corporal foram mais representativos. A obesidade é citada como fator predisponente para a RLCC no cão por promover carga excedente em um ligamento que já pode estar comprometido pelo processo degenerativo. ${ }^{2,4}$ Neste estudo, apesar de sabermos que grande parte dos cães recebia ração comercial, não tivemos a informação sobre qual o tipo e nem se os animais encontravam-se ou não acima do peso ideal. Se avaliarmos a freqüência de RLCC quanto ao intervalo de peso corpóreo, notamos que esta foi maior em cães de 6 a $15 \mathrm{~kg}(32,82 \%)$ e entre 36 e $45 \mathrm{~kg}$ (17,03\%), o que pode estar mais relacionado às raças (Poodle e Rottweiler, por exemplo), e não propriamente, com o excesso de peso.

Segundo Piermattei, Flo e Decamp ${ }^{8}$, $30 \%$ a $40 \%$ dos cães com RLCC apresentarão ruptura do ligamento contralateral em um período de 2 anos. Bennett et al. ${ }^{3}$ observaram 31\% de RLCC bilateral em 111 cães estudados. Neste estudo $12,38 \%(n=40)$ dos animais apresentaram RLCC bilateral, diagnóstico não necessariamente realizado durante um mesmo exame clinico e estando abaixo do valor relatado pelo autor acima. Talvez este valor esteja subestimado, pois devido à evolução muitas vezes insidiosa da doença o diagnóstico não tenha sido feito. Concordamos com Doverspike et al. ${ }^{18}$, sobre a importância do exame radiográfico do joelho contralateral a fim de descartar sinais de doença articular degenerativa, a qual poderia sugerir um provável diagnóstico de ruptura parcial ou total do ligamento cruzado cranial.

Apesar da etiopatogenia da RLCC ser ainda obscura, achados importantes têm sido obtidos em estudos realizados 10,14,19,20 Diferentes propriedades físicas do ligamento cruzado cranial entre as raças ${ }^{14}$, ângulo de platô da tíbia ${ }^{19}$, conformação dos membros pélvicos; hiper extensão da articulação do joelho ${ }^{10}$, além de estenose do sulco intercondilar ${ }^{20}$ têm sido imputados como prováveis fatores predisponentes para 
afecção. A correlação destas alterações com os vários padrões raciais poderiam fornecer informações importantes sobre a doença e, conseqüentemente, a sua prevenção e tratamento mais adequado.

\section{Conclusão}

Por meio deste estudo pode-se observar que os dados em nosso meio se assemelham com a literatura estrangeira, existindo tendência para a ocorrência da
RLCC em animais jovens de raças de grande porte e gigantes, o Rottweiler uma raça em comum e fêmeas mais representativas do que os machos. Porém, as raças de porte pequeno, bem como animais com peso entre 5 e $15 \mathrm{~kg}$ ainda continuam a ser bastante representativos. Acreditamos que estes dados são importantes para situar os padrões de ocorrência da RLCC no Brasil dentro da literatura estrangeira vigente, haja vista a alta freqüência da doença.

\title{
Epidemiologic retrospective study of dogs bearing cranial cruciate ligament rupture: 323 cases (1999 a 2005)
}

\begin{abstract}
The aim of this research was to characterize in the canine population the risk factors for cranial cruciate ligament rupture, which are breed, age, sex and body weight. The data base consisted of collected information from medical records of 323 dogs bearing cranial cruciate ligament rupture attended during a period of 7 years (1999 to 2005) in the Small Animal Surgical Service of the Veterinary Hospital of the Veterinary Medicine Faculty at São Paulo University. The results showed that the most prevalent breed were Leonberger $(100 \%)$, Cane Corso $(66,7 \%)$, Dogue de Bordeau (50\%), Starffordshire Terrier (40\%) e o Chow Chow (36\%). Also, Rottweiller $(11,16 \%)$ and Labrador Retriever $(8,1 \%)$ occupied the $10^{\circ}$ e $13^{\circ}$ positions, respectively. The ages $2,4,3,7$ and 6 (mean $=5,58)$ years old had the highest prevalence of cranial cruciate ligament rupture. Female $(59,14 \%)$ were more represented than males $(40,86 \%)$ and sexually intact animals $(76,15 \%)$ were more represented than neutered dogs $(17,76 \%)$. The group $f$ dogs weighing between 6 and $15 \mathrm{~kg}$ and between 36 and $45 \mathrm{~kg}$ showed the highest incidence. In this research was observed that the database of the risk factors for the dogs bearing cranial cruciate ligament rupture examined were similar to the that seen in the foreign literature. There is a tendency for rupture of ligament in an earlier age, large breeds and giant dogs. The Rottweiler was a common breed among our study and that of the foreign literature. Also, female were more represented than male dogs.
\end{abstract}

\section{Referências}

1 ARNOCZKY, S. P. Surgery of the stifle. The cruciate ligaments. Compendium on Continuing Education, $v$. 2, n. 2, p. 106-116, 1980.

2 VASSEUR, P. B. Stifle joint. In: SLATTER, D. Textbook of small animal surgery. $3^{\text {rd }}$. ed. Philadelphia: Saunders, 2003. p. 2090-2132.

3 BENNETT, D. et al. A reappraisal of anterior cruciate
Key words:

Cranial cruciate ligament. Knee.

Dog.

Osteoarthrosis. ligament disease in the dog. Journal of Small Animal Practice, v. 29, n. 5, p. 275-297, 1988.

4 DUVAL, J. M. et al. Breed, sex, and body weight as risk factors for rupture of the cranial cruciate ligament in young dogs. Journal of American Veterinary Medical Association, v. 215, n. 6, p. 811-814, 1999.

5 LAMPMAN, T. J.; LUND, E. M.; LIPOWITZ, A. J. Cranial cruciate disease: current status of diagnosis, surgery, and risk for disease. Veterinary Comparative 
Orthopedics and Traumatology, v. 16, n. 3, p. 122126, 2003.

6 WHITEHAIR, J. G.; VASSEUR, P. B.; WILLITS, N. H. Epidemiology of cranial cruciate ligament rupture in dogs. Journal of American Veterinary Medical Association. v. 203, n. 7, p. 1016-1019,1993.

7 VASSEUR, P. B. Clinical results following nonoperative management fo rupture of the cranial cruciate ligament in dogs. Veterinary Surgery, v. 13, n . 4, p. 243-246, 1984.

8 PIERMATTEI, D. L.; FLO, L.; DECAMP, C. E. The Stifle joint. In: PIERMATTEI, D. L.; FLO, L.; DECAMP C. E. Handbook of small animal orthopedics and fracture repair. $4^{\text {th }}$. ed. St Louis, Missouri: Saunders Elsevier, 2006. p. 562-632.

9 ROUSH, J. C.; HOHN, R. B.; DE ANGELIS, M. Evaluation of transplantation of the long digital extensor tendon for correction of anterior cruciate ligament rupture in dogs. Journal of American Veterinary Medical Association, v. 156, n. 3, p. 309312, 1993.

10 LEWIS, L. D.; MORRIS JR, M. L.; HAND, M. S. An evaluation of 62 cases of cruciate rupture stabilized using the modified tendon transfer technique. Veterinary Record, v. 94, n. 2, p. 32-38, 1974.

11 VASSEUR, P. B.; POOL, R. R.; ARNOCZKY, S. P. Correlative biomechanical and histologic study of the cranial cruciate ligament in dogs. American Journal of Veterinary Research, v. 46, n. 9, p. 1842-1854, 1985.

12 WINGFIELD C. et al. Comparision of the biomechanical properties of Rottweiler and racing greyhound cranial cruciate ligaments. Journal of Small
Animal Practice, v. 41, p. 303-307, 2000.

13 HARASEN, G. Canine cranial cruciate ligament rupture in profile. Canine Veterinary Journal, v. 44, n. 5, p. 845-846, 2003.

14 BARNES, A. J. Rupture of the anterior ligament of the dog; a survey from practices in the kent region BSAVA. Journal of Small Animal Practice, v. 18, n . 2, p. 55-59, 1977.

15 GAMBARDELLA, P. C.; WALLACE, L. J.; CASSIDY, F. Lateral suture technique for management of anterior cruciate ligament rupture in dogs: a retrospective study. Journal of American Veterinary Medical Association, v. 178, n. 1 , p. 33-38, 1981.

16 SMITH, G. K.; TORG, J. S. Fibular head transposition for repair of cruciate deficient stifle in the dog. Journal of American Veterinary Medical Association, v. 187, n. 4, p. 375-383, 1985.

17 SHIKATA, J.; SANADA, H.; YAMAMOTO, T. Experimental studies of the elastic fiber of the capsular ligament: influence of aging and sex hormones on the hip and knee joint capsule of rats. Connect Tissue Research, v. 7, n. 1, p. 21-27, 1979.

18 DOVERSPIKE, M. et al. Contralateral cranial cruciate ligament rupture: Incidence in 114 dogs. Journal of American Veterinary Medical Association, v. 29, n. 2, p. 167-170, 1993.

19 READ, R. A. Deformity of the proximal tibia in dogs. Veterinary Record, v. 111, n. 13, p. 295, 1982.

20 AIKEN, S. W.; KASS, P. H.; TOOBS, J. P. Intercondylar notch width in dogs with and without cranial cruciate ligament injuries. Veterinary Comparative Orthopedics and Traumatology, v. 8, n. 3 p. 128-132, 1995. 